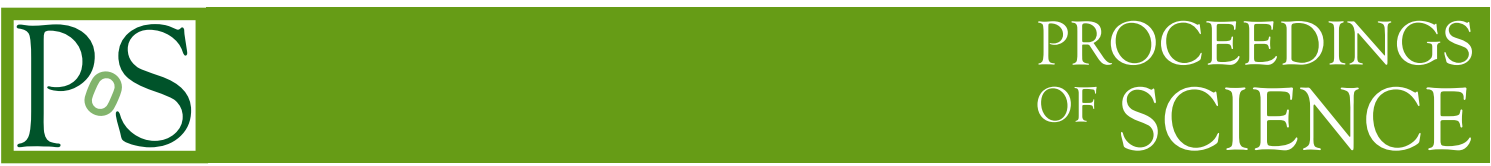

\title{
The enviroment of Low Surface Brightness Galaxies
}

\author{
Stefan Dominik Rosenbaum* \\ AIRUB, Germany \\ E-mail: roseneastro.rub.de
}

Dominik J. Bomans

AIRUB, Germany

E-mail: bomanseastro.rub.de

The formation and evolution scenarios that led to the existence of Low Surface Brightness (LSB) galaxies are not well understood yet. Although LSBs have HI components with low surface densities (systematically below the Kennicutt criterion), they can be regarded as gas-rich in general. Hence, the key in understanding LSBs lies in the answer to the question what prevented them from sufficient star formation. Using the public data releases of the Sloan Digital Sky Survey (SDSS), we investigated the galaxy density around LSBs in comparison to High Surface Brightness (HSB) galaxies. We performed a number counting analysis in three dimensions within spheres of several radii on a sample of HSBs and LSBs with well measured SDSS redshifts. On scales between 2 and $5 \mathrm{Mpc}$ our results show significantly lower galaxy densities in the vicinity of LSBs compared to HSBs. At larger scales than $5 \mathrm{Mpc}$ LSBs and HSBs share the same clustering properties but on scales below $2 \mathrm{Mpc}$ the galaxy densities in the neighbourhood of LSBs lie systematically (but only with a slight significance) below that of HSBs. In the pie slice diagrams LSBs favour the outer rims of the filaments of the Large Scale Structure and some LSBs are even found in voids. These results give strong evidence to a formation and evolution scenario where LSBs were formed in low density environments. Hence, the lack of tidal interactions with companions must have caused the absence of an effective trigger for a sufficient starburst.

Baryons in Dark Matter Halos

5-9 October 2004

Novigrad, Croatia

* Speaker. 

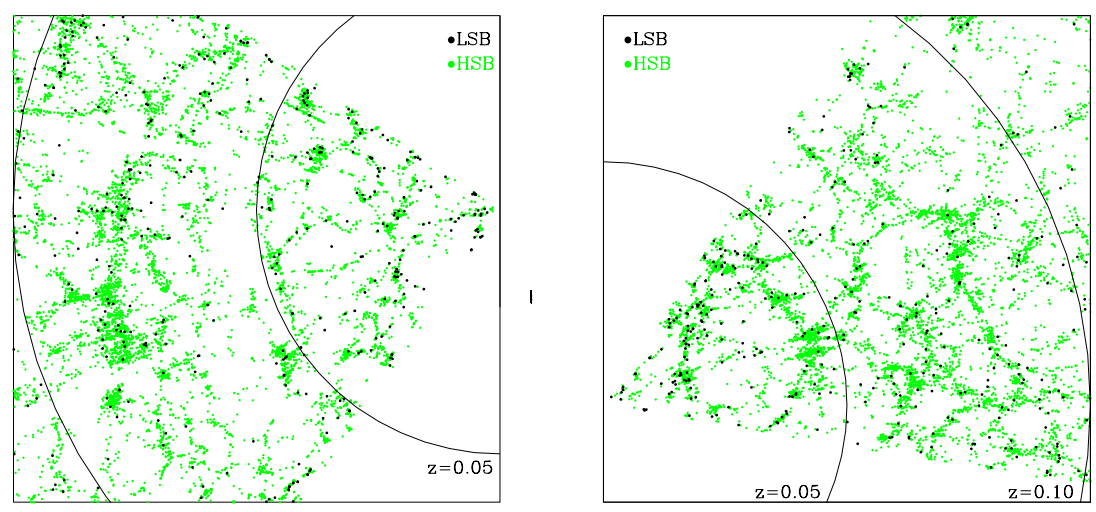

Figure 1: The two analysed pie slices. The right ascension sectors $147^{\circ} \leq \alpha \leq 233^{\circ}$ (left) and $354^{\circ} \leq \alpha \leq$ $53^{\circ}$ (right) are plotted containing a total of $\sim 800$ LSB galaxies (black dots) and $\sim 16000$ HSB galaxies (green/grey dots).

\section{Introduction}

The existence of gas-rich disk-galaxy-like LSB galaxies with central surface brightnesses of $\mu_{B}>22.5 \mathrm{mag} / \operatorname{arcsec}^{2}$ has been established over the last 15 years, whereas the formation and evolution scenarios that led to such a class of galaxies with a sparse stellar population are not well understood so far (e.g., [3]). Although LSB galaxies have HI components with low surface densities (e.g., [11]) systematically below the Kennicutt [4] criterion for star formation, they can be regarded as gas-rich in general (e.g., [6]).

Therefore, the key in understanding LSB galaxies lies in the answer to the question what prevented them from suffi cient star formation. One way to reconstruct the evolutionary history and to understand the properties of these galaxies might be found in the nature of the small- and largescale environments in which LSB galaxies are embedded, since the lack of star formation can only occur if the galaxies were formed in low density regions. Only a low density scenario can warrant that neither tidal encounter with companions nor infall of massive gas clouds could have taken place and could have triggered a suffi cient star formation which would have gradually brightened the stellar disk. Evidence for a stronger isolation of LSB galaxies in comparison to HSB galaxies was found before by [1] and [5]. A lack of nearby $(r \leq 0.5 \mathrm{Mpc})$ companions of LSB galaxies was detected by [12]. Today, with the availability of several substantial galaxy redshift surveys, the possibility for intensive studies on the environmental galaxy densities of LSB galaxies is given.

\section{Data Analysis and Results}

We retrieved LSB candidates and a HSB comparison sample from the Early Data Release (EDR) of the Sloan Digital Sky Survey (SDSS, [10]). The sample was limited to the two equatorial stripes of the EDR and to a redshift of $0.02 \leq z \leq 0.1$. In order to minimize the uncertainty of the redshift, a z-confi dence greater than $90 \%$ was demanded. A sample of 16123 galaxies was obtained. We distinguished between LSB galaxies with a central surface brightness $\mu_{B}>22.5 \mathrm{mag} / \mathrm{arcsec}^{2}$ 

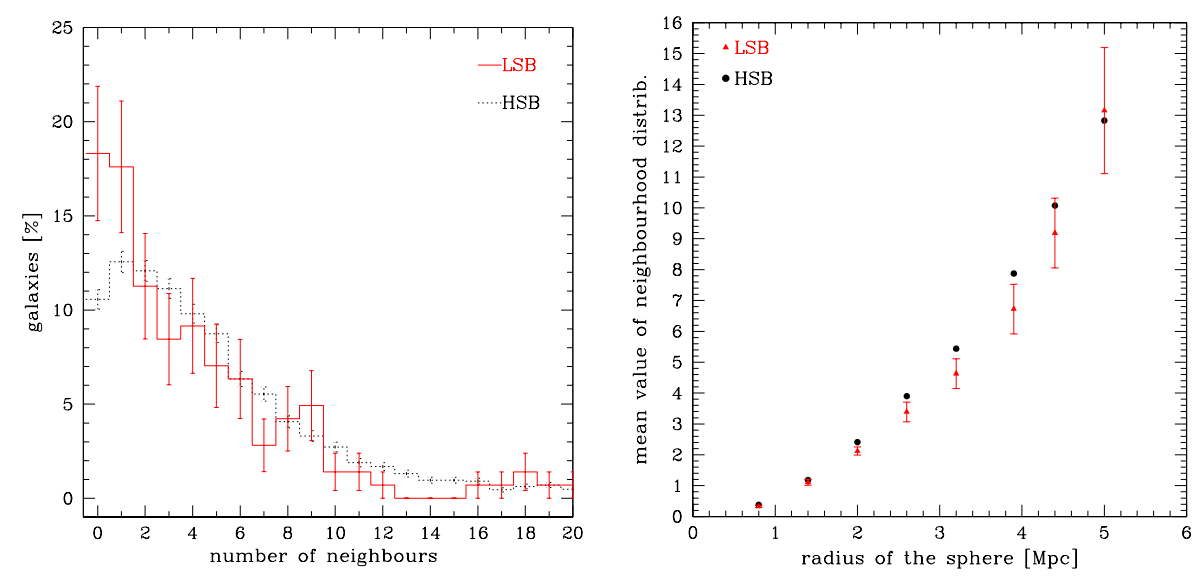

Figure 2: The left panel shows the distribution of the number of neighbours within a sphere with a radius of $r=3.2 \mathrm{Mpc}$ for LSB (solid line) and HSB (dashed line) galaxies. The right panel contains the mean values of the neighbourhood distributions for LSB (triangle) and HSB galaxies (dots) at several sphere radii.

and HSB galaxies. The central surface brightness $\mu_{B}$ was calculated for each galaxy from the central annulus $\left(0.23^{\prime \prime}\right)$ of the radial surface brightness profi le in the SDSS-fi lters $g$ and $r$ using an equation following [8]. With these selection criteria a sample of 804 LSB galaxies from both equatorial stripes of the EDR with a redshift of $z \leq 0.1$ was obtained. In this data set 15319 Galaxies remained as HSB galaxies.

The environmental density for each LSB and HSB galaxy of the sample was measured by counting the number of galaxies within a sphere around the scrutinised galaxy. First the code converted the redshift, right ascension and declination of all galaxies from our sample into the three dimensional spatial distribution of galaxies. Therefore a Hubble constant of $H_{0}=71 \mathrm{~km} / \mathrm{s}$ ([9]) was used. The purpose of the algorithm was to deliver the number of neighbours within a sphere of a certain radius for every single galaxy. The radius of the sphere was a fi xed parameter during each run. In several runs the radius of the sphere was varied between $r=0.8 \mathrm{Mpc}$ and $r=8.0 \mathrm{Mpc}$ in steps of $0.6 \mathrm{Mpc}$. In order to avoid biasing of the sample due to boundary effects, an edge correction was applied. For a detailed description of the data analysis see [7].

The pie slice diagrams (Fig. 1) show that LSB galaxies are located in the fil laments of the Large Scale Structure (LSS) traced by the distribution of HSB galaxies. Further investigations of the plot lead to the impression that LSB galaxies show the tendency to be located at the edges of these fi laments more often than in the center. Some LSB galaxies are even found in void regions.

The results of the statistical analysis using number counting within spheres were plotted in a diagram for each sphere radius showing the neighbouring statistics for LSB and HSB galaxies at several scales. Figure 2 shows as an example the distribution of the number of neighbours within a sphere of the radius $r=3.2 \mathrm{Mpc}$ (left panel) around each HSB and LSB galaxy. The mean values of neighbours for LSB and HSB galaxies were calculated for every applied sphere radius and are displayed in the right panel as a function of the corresponding radius. On lower scales (with $r=2.0 \mathrm{Mpc}$ and below, Fig. 2 right panel) the environmental statistics of the LSB galaxy distribution follows the distribution generated by the HSB galaxies within error margins as well. However, all LSB mean values lie systematically (but partially not signifi cantly) below 
the corresponding HSB value. Studies of the environment at larger scales $(2.0 \leq r \leq 5.0 \mathrm{Mpc})$ led to different results. While at small scales $(r \leq 2.0 \mathrm{Mpc})$ the percentage of LSB galaxies with no or one neighbour is nearly the same as for HSB galaxies, this fact does not apply at intermediate scales $(2 \leq r \leq 5 \mathrm{Mpc}$, Fig. 2, left panel). This result is reproduced by the dependency of the mean values on the sphere radius (right panel, Fig. 2). On scales between 2 and $4 \mathrm{Mpc}$ the mean values are signifi cantly lower for the LSB galaxies than for the HSB sample. For $2.6 \leq r \leq 4 \mathrm{Mpc}$ a Kolmogorov-Smirnov test rejects the null hypothesis that the distributions for LSB and HSB neighbours are the same at a confi dence level greater than $92 \%$ with a maximum value of $99.3 \%$ at a radius of $2.6 \mathrm{Mpc}$. For further details see [7].

\section{Summary and Conclusions}

Figure 1 shows that the spatial distribution of LSB galaxies follows in general the LSS defi ned by HSB galaxies which is in good agreement with the results from investigations of LSB galaxies in the Century Survey ([2]). However, the LSB galaxies seem to favour the outer parts of the fi laments and there is a handful of extremely isolated LSB galaxies located in voids of the LSS. The statistical results show that the isolation of LSB galaxies takes place on intermediate scales beyond the size of compact groups but in the range of the size of large groups and LSS fi laments as well. For smaller $(r \leq 2 \mathrm{Mpc})$ and larger scales $(r \geq 5 \mathrm{Mpc})$ no signifi cant differences in the statistical environments could be found in this study.

Based on our results presented here, we conclude that the galaxies formed in voids of the LSS and have drifted to the outer parts of the fil laments. This scenario is discussed extensively in [7]. The isolation of LSB galaxies on intermediate and small scales must have effected their evolution since tidal encounters acting as triggers for star formation would have been rarer in these LSB galaxies than in HSB galaxies.

\section{References}

[1] Bothun, G. D., Schombert, J. M., Impey, C. D., Sprayberry, D. \& McGaugh, S. S. 1993, AJ, 106, 530

[2] Brown, W. R., Geller, M. J., Fabricant, D. G., \& Kurtz, M. J. 2001, AJ, 122, 714

[3] Impey, C., \& Bothun, G. 1997, ARAA, 35, 267

[4] Kennicutt, R. C. 1998, ARAA, 36, 189

[5] Mo, H. J., McGaugh, S. S. \& Bothun, G. D. 1994, MNRAS, 267, 129

[6] Pickering, T. E., Impey, C. D., van Gorkom, J. H., \& Bothun, G. D. 1997, AJ, 114, 1858

[7] Rosenbaum, S. D., \& Bomans, D. J. 2004, A\&A, 422, L5

[8] Smith, J. A., Tucker, D. L. et al. 2002, AJ, 123, 2121

[9] Spergel, D. N., Verde, L. et al. 2003, APJS, 148, 175

[10] Stoughton, C., Lupton, R. H. et al. 2002, AJ, 123, 485

[11] van der Hulst, J. M., Skillman, E. D., Smith, T. R., Bothun, G. D., McGaugh, S. S., \& de Blok, W. J. G. 1993, $A J$, 106, 548

[12] Zaritsky, D., \& Lorrimer, S. J. 1993, in: Evolution of Galaxies and their Environment, 82 